\title{
Diagnostique de production de l'oignon dans la partie Septentrionale du Cameroun
}

\author{
Jérémie DINISSIA ${ }^{1}$, Lucien TATCHUM TCHEUNTEU ${ }^{*}$, Abba MAIMOUNA ${ }^{1}$, \\ HAWAOU $^{2}$, Habib ALIUM ${ }^{1}$, Dieudonné NWAGA ${ }^{2}$ et Clautide MEGUENI ${ }^{1}$ \\ ${ }^{1}$ Laboratoire de Biodiversité et de Développement Durable, Département de Sciences Biologiques, \\ Université de Ngaoundéré, BP 454, Ngaoundéré, Cameroun. \\ ${ }^{2}$ Centre de Biotechnologies, Laboratoire de Microbiologie des sols, Université de Yaoundé I, \\ BP 812, Yaoundé, Cameroun. \\ *Auteur correspondant ; E-mail : tatchumlucien@yahoo.fr ; Tél.00 237699145141
}

\section{RÉSUMÉ}

Oignon (Allium cepa L.) est une culture maraîchère présentant un atout économique important pour les paysans Camerounais. Cette étude visait à faire un diagnostic de production de l'oignon dans la partie Septentrionale du Cameroun afin de développer des stratégies d'amélioration durable de cette filière. Une enquête a été menée pour déterminer le profil des acteurs de la filière oignon, les variétés cultivées, les techniques culturales ainsi que les contraintes de production. Les enquêtes s'étaient déroulées en 2017 auprès des acteurs de la filière oignon. L'échantillonnage était constitué de 60 personnes dans chacune des trois régions du Grand Nord Cameroun, soit un échantillonnage global de 180 personnes. Les résultats montrent que la plupart des acteurs sont des producteurs et sont 1,47 fois plus nombreux que les commerçants. 04 variétés d'oignon sont cultivées : Goudami, Chagari, Belami et violet de Galmi. La variété Goudami avait un rendement en bulbe élevé (10 à 35 t/ha), une durée de conservation des bulbes plus importante et est la plus cultivée. La quasi-totalité (69\%) des producteurs utilise les engrais chimiques pour améliorer le rendement de cette culture. Ces informations serviront de base de données dans le programme de perfectionnement de la filière oignon au Cameroun.

(C) 2021 International Formulae Group. All rights reserved.

Mots clés : Oignon, acteurs, production, Cameroun.

\section{Diagnosis of onion production in the Northern Part of Cameroon}

\begin{abstract}
Onion (Allium cepa L.) is a market garden crop that is a major economic asset for Cameroonian farmers. This study aimed at diagnosing onion production in the Northern part of Cameroon in order to develop strategies for onion sustainable improvement. A survey was conducted to determine the actors profile relative to onion
\end{abstract}


sector, varieties grown, crop techniques as well as production constraints. The surveys were conducted in 2017 among actors of onion sector. The sample consisted of 60 people in each of the three regions of Far North Cameroon, for an overall sample of 180 people. The results shown that most of the actors are producers, they are 1.47 folds greater than traders. Four onion varieties are grown in the Northern part of Cameroon: Goudami, Chagari, Belami and Galmi violet. The Goudami variety presents high bulb yield (10 to $35 \mathrm{t} / \mathrm{ha}$ ) and longer bulb shelf life, and is the most widely cultivated. Almost all (69\%) of the producers use chemical fertilizers to improve onion growth. This information will be used as a database to improve the performance of onion sector in Cameroon.

(C) 2021 International Formulae Group. All rights reserved.

Keywords: Onion, actors, production, Cameroon.

\section{INTRODUCTION}

Allium cepa L. est une plante herbacée bisannuelle vivace de la famille des Alliaceae (Fritsch et Friesen, 2002; Abdou, 2014; Boukeria, 2017). Cette plante est depuis son adoption cultivée pour ses vertus médicinales et alimentaires. Sur le plan médicinal, l'oignon est utilisé pour le traitement des infections, inflammations respiratoires et rhumatismes, ses propriétés hypolipédemiantes sont importantes pour la prévention des maladies cardiovasculaires (Tache et al., 2007). Sur le plan alimentaire, l'oignon est riche en éléments nutritifs tels que les glucides, protides, lipides, sels minéraux ( $\mathrm{Ca}, \mathrm{Mg}, \mathrm{K}, \mathrm{Fe})$, ainsi qu'en multiples vitamines (C, B, E) (Konate et al., 2018). Une partie des feuilles, avant la formation du bulbe est mangée crue, seule ou en salade (Cathala et al., 2003 ; Konate et al., 2018). L'oignon est classé deuxième au rang des légumes les plus cultivés au monde après la tomate (Abdou, 2014). La production mondiale de cette culture maraîchère s'élevait à 82,85 $10^{6}$ tonnes par an avec la Chine, l'Inde et les Etats Unis comme les trois premiers pays producteurs. En Afrique, les principaux pays producteurs d'oignon sont l'Egypte, l'Algérie, le Maroc et le Nigéria (FAOSTAT, 2013).

Dans la sous-région Afrique centrale en particulier, la production annuelle d'oignon est d'environ 90000 tonnes avec un rendement moyen de $10 \mathrm{t} / \mathrm{ha}$. Cette production dans les régions du Nord et de l'Extrême-Nord, Cameroun représente $85 \%$ de la production nationale (Cathala et al., 2003 ; Kamga et al., 2016). L'oignon est classé troisième culture de rente après le coton et l'arachide et est la première spéculation maraîchère dans la partie Septentrionale du pays (Cathala et al., 2003). La filière oignon est un poumon économique qui regroupe les différents acteurs et l'essentiel de la production nationale (Maldangoï et al., 2003). Dans les exploitations agricoles en culture intensive de la région de l'ExtrêmeNord, l'oignon représente $45 \%$ du revenu net (Madi, 2000). Dans la zone de production, les appuis techniques et financiers restent ponctuels et les moyens producteurs d'oignons souffrent d'un accès très limité aux crédits de campagne (pour les intrants) (Cathala et al., 2003 ; PADFA, 2010), ce qui serait à l'origine de la faible production d'oignon observée au Cameroun. C'est ainsi que plusieurs travaux (FAO, 2011 ; Mamadou, 2012) rapportent que la production d'oignon au Cameroun n'arrive pas toujours à satisfaire les besoins de la consommation nationale. A cet égard, le développement des stratégies d'amélioration durable de la disponibilité de l'oignon au Cameroun est une nécessité.

C'est ainsi que cette étude visait à faire un diagnostic de production de l'oignon dans la partie Septentrionale du Cameroun afin de mettre sur pied des stratégies d'amélioration durable de cette filière. Spécifiquement, il s'agit de: (1) faire une caractérisation des acteurs de la filière oignon dans la partie Septentrionale du Cameroun ; (2) recenser les 
variétés d'oignons cultivées dans le Grand Nord Cameroun; (3) étudier les techniques culturales de cette spéculation ; (4) ressortir les difficultés rencontrées dans les divers circuits. L'intérêt et l'utilité de ce travail repose sur le fait que les résultats de cette étude serviront de base de données pour l'amélioration de la filière oignon.

\section{MATÉRIEL ET MÉTHODES \\ Description de la zone d'étude}

Les études se sont déroulées dans les 03 régions de la partie Septentrionale du Cameroun, plus précisément dans les localités de Salak, Ziling et Domayo à l'Extrême-Nord ; Lopéré, Djoulol à Pitoa et Touboro au Nord ; Toubaka (Dom, Wak) et Ngaoundéré II dans l'Adamaoua (Figure 1). La culture d'oignon est ancrée dans les mœurs des paysans de ces localités. Par ailleurs, cette culture maraîchère représente un atout économique important pour les populations du Grand Nord Cameroun (Maldangoï et al., 2003).

\section{Méthodologie}

\section{Plan expérimental et collecte des données}

Les données de cette étude ont été collectées au moyen d'une enquête et des explorations des champs paysans, magasins et marchés. Les enquêtes se sont déroulées de janvier 2017 à décembre 2017 auprès des producteurs, commerçants et agents des structures de recherche. L'échantillonnage était constitué de 60 personnes dans chacune des régions de l'Adamaoua, du Nord et de
l'Extrême Nord, soit un échantillonnage global de 180 personnes. L'interview était semistructurée et individuelle. Le questionnaire préalablement élaboré comportait des questions ouvertes, fermées et d'autres orientées. Les questions ouvertes permettaient aux personnes interrogées de donner leur opinion par rapport à une question posée. Les questions fermées quant à elles ne lui accordaient que le choix de répondre par oui ou non. En ce qui concerne les questions orientées, une série de proposition étaient faites au répondant, qui est appelé à choisir une ou plusieurs des propositions. Les grandes rubriques abordées dans la fiche d'enquête portaient sur la caractérisation socioéconomique des acteurs de la filière oignon; le recensement des différentes variétés d'oignons cultivées et l'étude des techniques de cultures ainsi que de conservation de cette spéculation ; les difficultés rencontrées par les acteurs. Les observations directes consistaient à déterminer les caractéristiques physiques (couleur) des variétés d'oignon cultivées et de relever les difficultés rencontrées.

\section{Analyse statistique des données}

Les données collectées ont été statistiquement analysées grâce au logiciel «Statgraphics plus version 5.0», qui effectue l'analyse de variance (ANOVA). Le test de Duncan est utilisé pour déterminer la différence entre les moyennes des différents traitements. Le logiciel Microsoft Excel version 2016 a été utilisé pour calculer le pourcentage et tracer les diagrammes. 


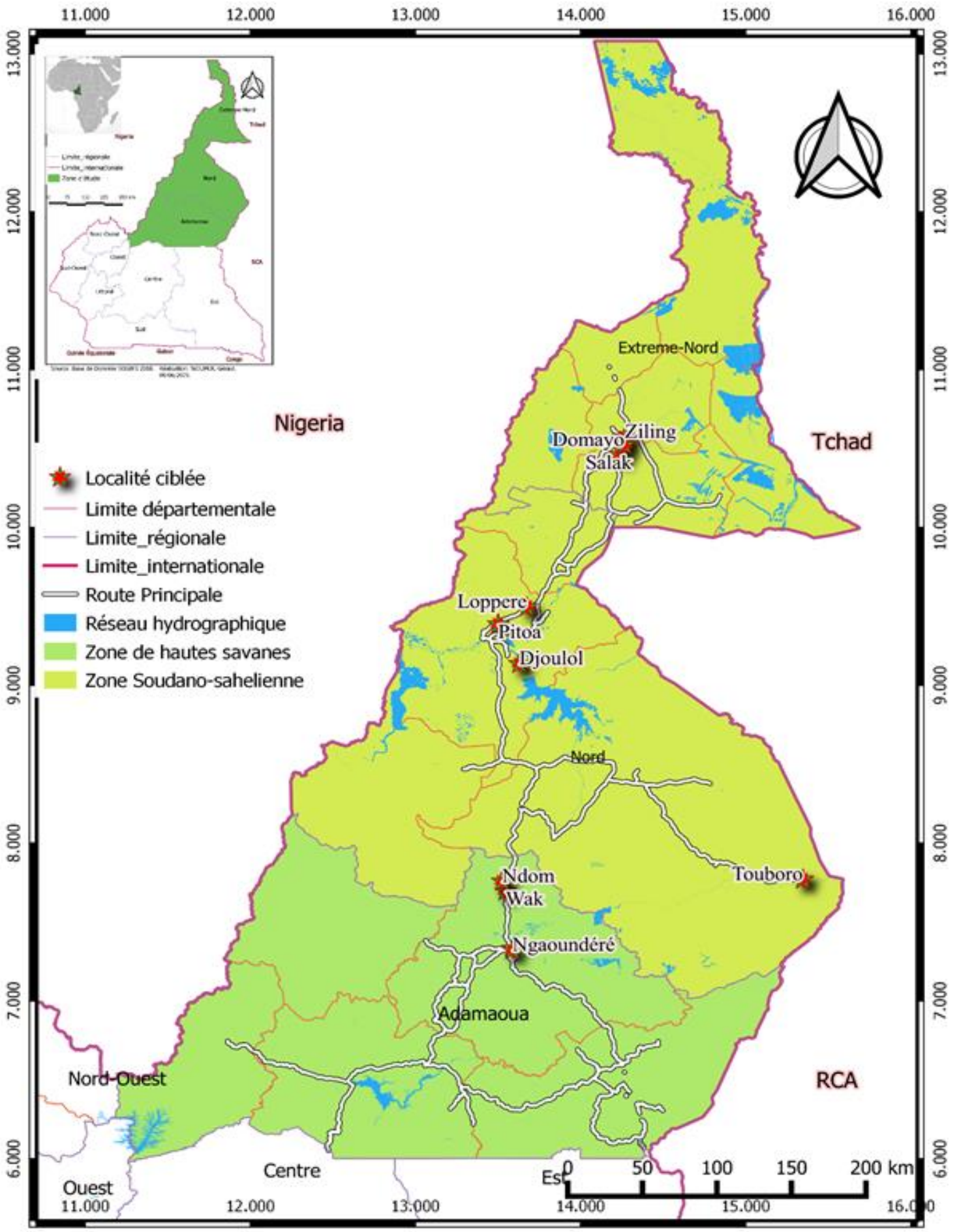

Source: Base de Données SOGEFI 2018.

Figures 1 : Positions des sites d'enquêtes sur la carte de la partie Septentrionale du Cameroun. 


\section{RÉSULTATS}

Profil des acteurs de la filière oignon dans la zone Septentrionale du Cameroun

\section{Proportion des acteurs}

Dans le Grand Nord Cameroun, deux types d'acteurs sont impliqués dans la filière oignon, les producteurs et commerçants (Tableau 1). Globalement, Il n'existe pas de différence significative entre les proportions des acteurs de la filière oignon dans les trois régions de la partie Septentrionale du Cameroun. Cependant, les proportions des producteurs varient de $23,33 \%$ dans la région de l'Adamaoua Cameroun à 78,33\% dans le Nord Cameroun. Par contre la proportion des commerçants varie de $21,67 \%$ dans le Nord Cameroun à $76,67 \%$ dans la région de l'Adamaoua Cameroun. Globalement, les producteurs représentent la proportion la plus élevée $(59,44 \pm 31,28 \%)$ des acteurs de la filière oignon par rapport à celle des commerçants $(40,56 \pm 31,28 \%)$. La proportion des producteurs d'oignon du Nord Cameroun est plus élevée que celle de l'Adamaoua et de l'Extrême Nord. Cette proportion est 3,36 fois plus élevée dans le Nord que dans l'Adamaoua. Âges des acteurs

La Figure 2 illustre les proportions des acteurs de la filière oignon en fonction des tranches d'âges dans la partie Septentrionale du Cameroun. La proportion des acteurs varie significativement $(\mathrm{p}<0,05)$ en fonction de l'âge. Les tranches d'âges des acteurs sont comprises entre 15 et 55 ans. Toutefois, les acteurs de la filière oignon dont l'âge est compris entre 35 et 45 ans représentent la plus grande proportion des acteurs $(41,67 \pm 1,67 \%)$. Les acteurs les plus jeunes, c'est-à-dire ceux dont l'âge est compris entre 15 et 25 ans sont les moins représentés avec un pourcentage de $16,67 \pm 10,27 \%$. La plupart des acteurs de la filière oignon dans le Grand Nord Cameroun sont des adultes dont l'âge varie de 35 à 45 ans et ils sont 2,50 fois plus nombreux que les jeunes (15 et 25 ans) impliqués dans cette filière.

\section{Niveau d'éducation des acteurs}

Les acteurs de la filière oignon de la partie Septentrionale du Cameroun ont des niveaux d'éducation variable d'une région à l'autre (Tableau 2). Certains de ces acteurs sont analphabètes, d'autres ont un niveau primaire, secondaire ou universitaire. Les proportions des acteurs varient significativement $(p<0,05)$ en fonction du niveau d'étude. De façon globale, les acteurs de la filière oignon dont le niveau d'étude est secondaire sont les plus représentés $(49,44 \pm 12,62 \%)$ tandis que la proportion des acteurs ayant un niveau d'étude supérieure est la plus faible $(6,11 \pm 7,87 \%)$. Dans cette étude la proportion des acteurs avec un niveau d'étude secondaire est 3,87 fois plus élevée que celle des analphabètes et 8,09 fois plus élevée que celle des acteurs avec un niveau universitaire.

\section{Niveau d'expérience des acteurs}

Il apparait clairement que les acteurs de la filière oignon de la partie Septentrionale du Cameroun ont plusieurs années d'expérience dans cette filière (Tableau 3). Les analyses statistiques révèlent que les proportions des acteurs de la filière oignon ne varient pas en fonction de l'âge dans le Grand Nord Cameroun. Cependant, la proportion des acteurs ayant plus d'ancienneté dans la filière (5 ans et plus) est plus élevée dans le Nord Cameroun (78,33\%). Aucun acteur de l'Adamaoua n'a plus de 5 ans d'expérience dans la filière. De façon globale, le nombre d'acteurs ayant plus d'expérience dans la filière ( 5 ans et plus) est plus importante $(51,67$ $\pm 44,75 \%)$ tandis que la proportion des jeunes acteurs ([2-3 ans d'expérience [) est la plus faible $(17,22 \pm 21,75 \%)$. Dans cette étude, le nombre d'acteurs bien expérimenté dans la filière ( 5 ans et plus) est 3,00 fois plus élevé que le nombre de jeunes acteurs (2 à 3 ans d'expérience).

\section{Diagnostic de production de l'oignon dans le Grand Nord Cameroun Variétés cultivées}

La Figure 3 illustre la proportion des variétés cultivées en fonction des régions de l'Adamaoua Cameroun. Il ressort des analyses statistiques que les proportions des variétés cultivées varient significativement $(p<0,05)$ en 
fonction des régions du Grand Nord Cameroun. La variété Goudami est la plus cultivée dans chacune des régions de notre zone d'étude, tandis que la variété Balami est la moins cultivée dans les régions de l'Adamaoua et de l'Extrême Nord. La variété Chagari est la moins sollicitée par les producteurs d'oignon du Nord. La proportion des cultivateurs de la variété Goudami est la plus importante $(46,33 \pm 6,56 \%)$ tandis celle de la variété Belami est la plus faible $(9,44 \pm 5,09 \%)$. Les proportions des producteurs de la variété Violet de Galmi et Chagari sont respectivement de $24,99 \pm 7,26 \%$ et de $20,55 \pm 7,87 \%$. Le nombre des producteurs d'oignon de la partie Septentrionale du Cameroun qui s'intéressent à la variété Goudami est respectivement 4,91 fois ; 2,25 fois et 1,85 fois plus élevé que le nombre de producteurs des variétés Belami, Chagari et Violet de Galmi.

Les caractéristiques des variétés d'oignon cultivé dans le Grand Nord Cameroun sont consignées dans le Tableau 4. Il ressort de ce tableau que les bulbes des variétés Goudami et Belami sont de même couleur (Rose saumon), les couleurs des variétés Violet de Galmi sont respectivement violet et rouge (Figure 4). Parmi les 04 variétés d'oignon cultivées dans le Grand Nord Cameroun seule la variété Chagari est cultivée en saison sèche et pluvieuse. Les 03 autres variétés (Goudami, Violet de Galmi et Belami) ne sont cultivées qu'en saison sèche. Le rendement en bulbe de la variété Goudami est plus élevé (10 à $35 \mathrm{t} / \mathrm{ha}$ ) que ceux des autres variétés, la variété Chagari a la plus faible valeur du rendement en bulbe ( 10 à $20 \mathrm{t} / \mathrm{ha}$ ) et les variétés Violet de Galmi et Belami ont les mêmes rendements en bulbe (10 à $25 \mathrm{t} / \mathrm{ha}$ ). La conservation de la variété Goudami est plus aisée (4 à 6 mois) tandis que la durée moyenne de conservation de la variété Belami est la plus courte (3 à 4 mois). Il observé dans ce travail que la variété Goudami d'oignon a un rendement en bulbe élevé et la durée de conservation de ses bulbes est plus importante, ce qui justifierait l'intérêt de la plupart des producteurs d'oignon de la partie Septentrionale du Cameroun pour la culture de cette variété. A cet égard, il serait intéressant de développer des stratégies améliorantes de la productivité de la variété Goudami d'oignon dans le Grand Nord Cameroun.

Pratiques paysanne de culture d'oignon dans la partie Septentrionale du Cameroun Modes de préparation des champs de culture

Il ressort de la Figure 5 que les producteurs d'oignon du Grand Nord Cameroun utilisent les cassiers et les planche ou billon pour la culture d'oignon. La plupart (78\%) de ces producteurs forme les cassiers pour la culture de cette plante. La proportion des producteurs qui forme les cassiers pour la culture d'oignon est 3,55 fois plus élevée que ceux qui utilisent les billons. Les casiers sont des zones rectangulaires cultivées avec une légère profondeur tandis que les planches ou billons sont des zones rectangulaires cultivées avec une surélévation.

\section{Fertilisation des champs d'oignon}

Les producteurs d'oignon du Grand Nord Cameroun font recours aux engrais chimiques de synthèse (NPK, urée), amendements organiques (déchets d'animaux, composts) et biofertilisants pour améliorer les rendements de cette culture maraîchère (Figure 6). La quasi-totalité des producteurs d'oignon $(69 \%)$ utilise les engrais, alors que seulement $29 \%$ et $2 \%$ de ces producteurs améliorent la fertilité de leurs champs d'oignon respectivement à base des amendements organiques et des biofertilisants. Le nombre de producteurs qui utilisent les engrais chimiques est 2,55 fois plus élevé que le nombre de producteurs utilisant les engrais naturels.

\section{Difficultés de la filière oignon dans le Grand Nord Cameroun}

Les difficultés auxquelles font face les acteurs de de la filière oignon du Grand Nord Cameroun sont énormes. Au niveau de la production, les paysans n'ont pas toujours suffisamment de moyens financiers pour se 
procurer des semences et des engrais chimiques de synthèse qu'ils trouvent très coûteux. En plus l'oignon fait face à de nombreuses maladies dans la partie Septentrionale du Cameroun. Ces maladies sont: Botritys squamosa, Mildiou ou pourriture de collet, racines roses, pourriture blanche et pourriture basale. Le manque d'infrastructures appropriées pour la conservation (pourritures et la germination des bulbes) conduit à la vente précoce du produit. Concernant les difficultés liées à la commercialisation, il y'a un manque de marchés locaux dans plusieurs localités de notre zone d'étude, la chute du prix de l'oignon pendant la période de récolte propice. Au niveau du transport du produit dans les marchés interurbains, l'enclavement des localités de production rend exorbitant le coût de moyen de transport, mauvais état des routes.

Tableau 1 : Proportion des acteurs de la filière oignon dans la zone Septentrionale du Cameroun.

\begin{tabular}{lcc}
\hline Régions & \multicolumn{2}{c}{ Proportion des acteurs de la filière oignon (\%) } \\
\cline { 2 - 3 } & Producteurs & Commerçants \\
\hline Extrême Nord & 76,67 & 23,33 \\
\hline Nord & 78,33 & 21,67 \\
\hline Adamaoua & 23,33 & 76,67 \\
\hline Moyenne \pm Ecart type & $59,44 \pm 31,28^{\mathrm{a}}$ & $40,56 \pm 31,28^{\mathrm{a}}$ \\
\hline P-value & & \\
\hline
\end{tabular}

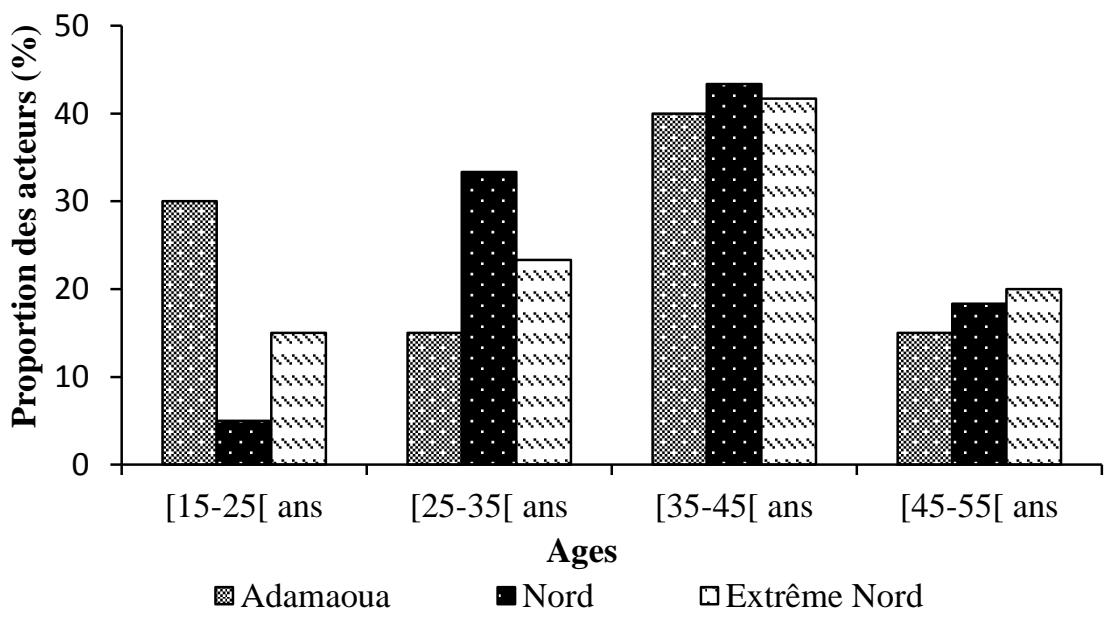

Figure 2 : Proportion (\%) des acteurs en fonction de l'âge. 
Tableau 2 : Proportion des acteurs de la filière en fonction des niveaux d'études.

\begin{tabular}{ccccc}
\hline \multirow{2}{*}{ Régions } & \multicolumn{3}{c}{ Proportion des acteurs en fonction du niveau d'étude (\%) } \\
\cline { 2 - 5 } & Analphabète & Primaire & Secondaire & Supérieur \\
\hline Adamaoua & 6,67 & 38,33 & 55 & 0 \\
\hline Nord & 13,33 & 25 & 58,34 & 3,33 \\
\hline Extrême Nord & 18,33 & 31,67 & 35 & 15 \\
\hline Moyenne \pm Ecart type & $12,77 \pm 5,84^{\mathrm{c}}$ & $31,67 \pm 6,65^{\mathrm{b}}$ & $49,44 \pm 12,62^{\mathrm{a}}$ & $6,11 \pm 7,87^{\mathrm{c}}$ \\
\hline
\end{tabular}

Tableau 3 : Proportion des acteurs de la filière oignon en fonction des années d'expérience.

\begin{tabular}{ccccc}
\hline Régions & \multicolumn{4}{c}{ Proportion des acteurs en fonction des années d'expérience (\%) } \\
\cline { 2 - 5 } & {$[\mathbf{2 - 3}$ ans [ } & {$[\mathbf{3 - 4}$ ans [ } & {$[\mathbf{4 - 5}$ ans [ } & [5 ans et plus [ \\
\hline Adamaoua & 41,67 & 43,33 & 15 & 0 \\
\hline Nord & 0 & 5 & 16,67 & 78,33 \\
\hline Extrême Nord & 10 & 5 & 8,33 & 76,67 \\
\hline Moyenne \pm Ecart type & $17,22 \pm 21,75^{\text {a }}$ & $17,77 \pm 22,12^{\text {a }}$ & $13,33 \pm 4,41^{\mathrm{a}}$ & $51,67 \pm 44,75^{\text {a }}$ \\
\hline
\end{tabular}

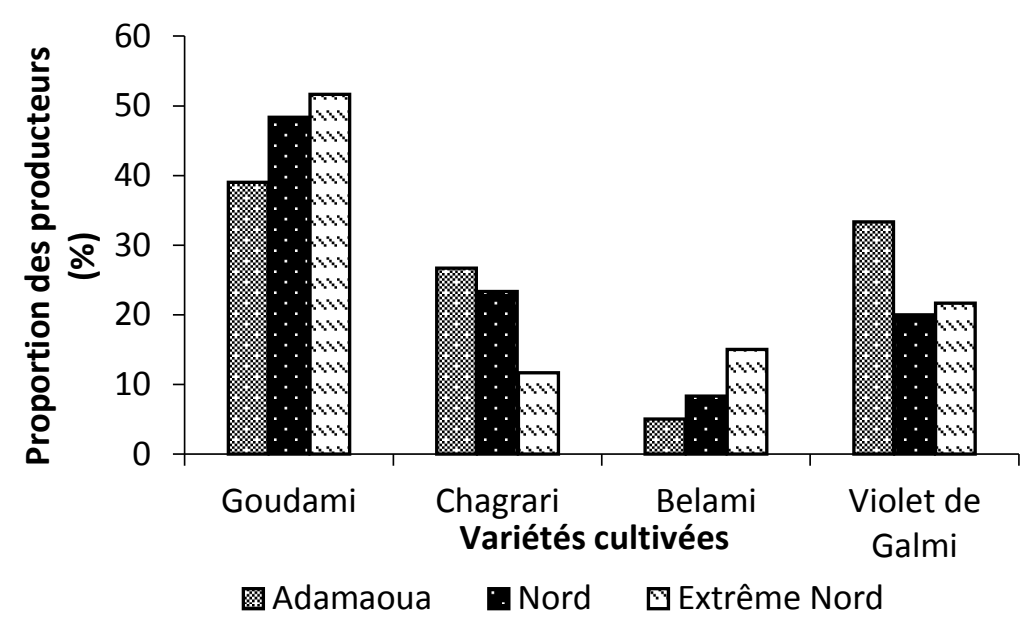

Figure 3 : Proportion des producteurs en fonction des variétés d'oignon.

Tableau 4 : Quelques caractéristiques des variétés d'oignon cultivées dans le Grand Nord Cameroun.

\begin{tabular}{lcccc}
\hline $\begin{array}{l}\text { Variétés } \\
\text { d'oignon }\end{array}$ & $\begin{array}{c}\text { Couleur du } \\
\text { bulbe }\end{array}$ & Saison de culture & $\begin{array}{c}\text { Rendement en bulbe } \\
\text { (t/ha) }\end{array}$ & $\begin{array}{c}\text { Durée de conservation } \\
\text { (mois) }\end{array}$ \\
\hline Goudami & Rose saumon ; & Sèche & $10-35$ & $4-6$ \\
\hline $\begin{array}{l}\text { Violet } \\
\text { Galmi }\end{array}$ & Violet & Sèche & $10-25$ & $4-5$ \\
\hline Chagari & Rouge & Sèche/pluvieuse & $10-20$ & $4-5$ \\
\hline Belami & Rose saumon & Sèche & $10-25$ & $3-4$ \\
\hline
\end{tabular}




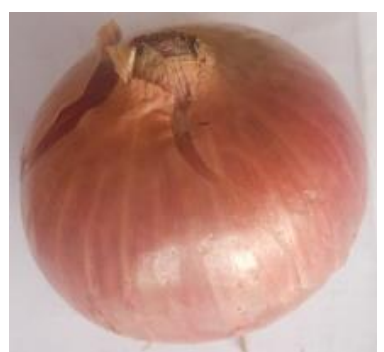

a) Goudami

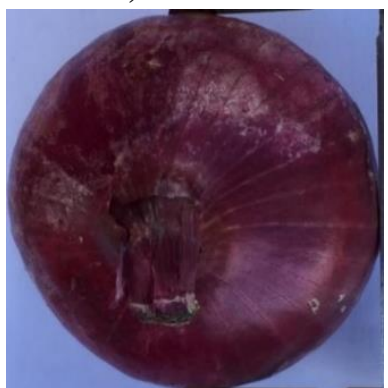

c) Violet de Galmi

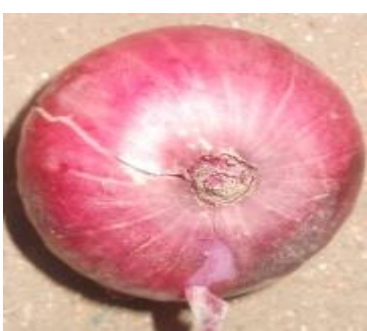

b) Chagari

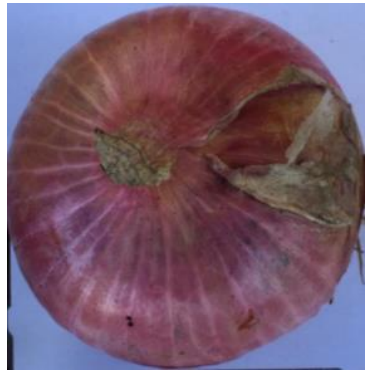

d) Belami

Figure 4 : Bulbes des variétés d'oignon cultivées présentant des couleurs diverses.

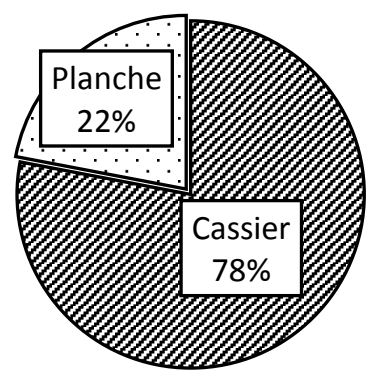

Modes de préparation des champs de culture

Figure 5: Proportion des producteurs d'oignon en fonction des modes de préparation des champs.

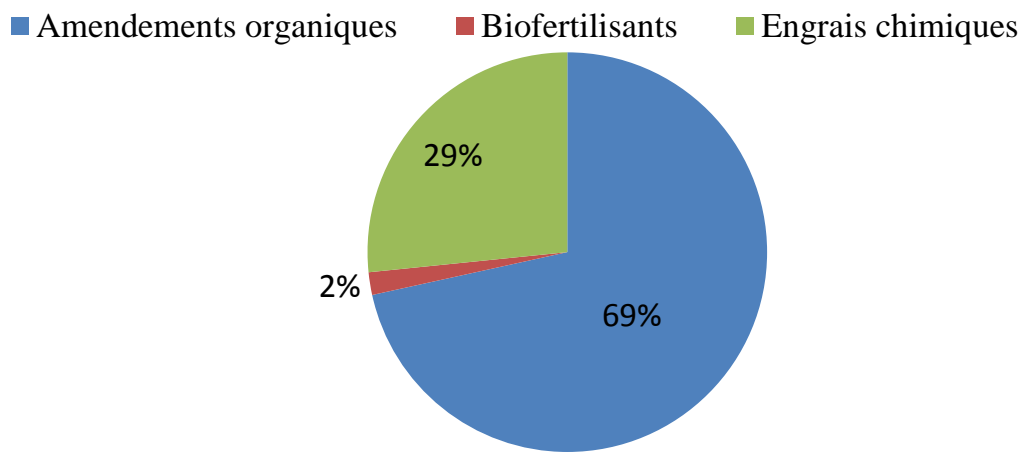

Figure 6 : Proportions des producteurs d'oignon en fonction de la fertilisation. 


\section{DISCUSSION}

Il a été observé dans cette étude qui visait à faire un diagnostic de production de l'oignon dans le Grand Nord Cameroun que les acteurs de la filière oignon dans le grand Nord Cameroun sont les producteurs et les commerçants, ce qui corrobore les données rencontrées dans la littérature. Les travaux de Mamadou (2012) sur l'appui à la commercialisation et la structuration de la filière l'oignon à l'Extrême Nord Cameroun ; de Folefack et Djouldé (2013) sur les stratégies de commercialisation des produits maraichers sur le marché de Maroua (Cameroun) révèlent que plusieurs acteurs interviennent dans la filière oignon à savoir les producteurs, les collecteurs villageois ou intermédiaires, les grossistes et les transporteurs. Les acteurs d'une filière représentent les maillons clés par lesquels passent des nouvelles techniques, ce qui suggère que les stratégies d'amélioration de la filière oignon qui pourraient être mises sur pied passeront par les producteurs et les commerçants. A cet égard, il serait intéressant d'étudier le profil de ces acteurs de la filière oignon (âge, niveaux d'étude) dans le Grand Nord Cameroun.

Les personnes dont les âges sont compris dans l'intervalle [35-45[ans sont fortement impliquées dans la filière oignon dans le Grand Nord Cameroun, ce qui corrobore les travaux de Sali et al. (2016) qui font un compte rendu de mission de diagnostic des causes de pourritures des oignons dans la région du Nord Cameroun et rapportent que $62 \%$ des acteurs sont des adultes. Par ailleurs une étude conduite par Kamga et al. (2016) sur l'inégalité du genre des exploitants dans la production d'oignon à l'Extrême Nord Cameroun révèlent que la majorité des exploitants agricoles de la filière oignon de l'Extrême Nord sont principalement âgés de 15 à 36 ans.

Il est constaté dans ce travail que les acteurs avec un niveau d'éducation secondaire sont fortement engagés dans la filière oignon, ce qui n'est pas en conformité avec les travaux de Kamga et al. (2016) qui rapportent que les proportions des acteurs avec un niveau d'étude primaire et secondaire impliquées dans la filière oignon dans le Grand Nord Cameroun sont respectivement de 49 et $25,50 \%$.

Le nombre d'années d'expérience des acteurs de la filière oignon du Grand Nord Cameroun varie de 2 à plus de 5 ans. Ce résultat corrobore en partie les données rapportées par Akoa et Mabou (2004); Kamga et al. (2016) qui trouvent que les différents acteurs impliqués dans la filière oignon ont une expérience de 10 ans dans la production avec une ancienneté d'au moins 3 ans dans la commercialisation. L'ancienneté des acteurs est un facteur de réussite car cela implique une bonne maîtrise de la filière afin de contourner les difficultés. Dans ce travail, les acteurs les plus anciens se trouvent à l'Extrême Nord, ceci se justifierait par le fait que la culture d'oignon est plus ancienne dans cette région du Cameroun que dans l'Adamaoua et le Nord. En effet, cette plante était d'abord introduite dans la région de l'Extrême Nord Cameroun avant de se répandre dans les autres régions du pays.

Les variétés d'oignon cultivées dans le Grand Nord Cameroun sont Goudami, Chagari, Belami et violet de Galmi., ce qui corrobore les données rencontrées dans la littérature. En effet, les travaux de Maldangoï et al. (2003) révèlent que la variété Goudami d'oignon est la plus produite au Nord Cameroun comparé aux variétés Violet de Galmi, Belami et Chagari. En outre, les travaux de Abdou et al. (2014) sur les systèmes de culture de l'oignon en Afrique, et ceux de Bassole et al. (2017) sur l'amélioration de la rentabilité du système de culture de l'oignon au Nord Cameroun rapportent que les principales variétés d'oignon cultivées sont Violet de Galmi, Goudami et Chagari. Il est observé dans cette étude que la variété Goudami d'oignon est la plus cultivée dans la partie Septentrionale du Cameroun, suggérant ainsi que cette variété d'oignon auraient un rendement en bulbes élevé.

La majorité des producteurs d'oignon du Grand Nord Cameroun utilise les engrais chimiques de synthèse pour la culture de 
l'oignon, ce qui corrobore les données rencontrées dans la littérature. Les travaux de plusieurs auteurs (Megueni et al., 2011; Kamga et al., 2016 ; Tchuenteu et al., 2018 ; Kamdem et al., 2020) rapportent que les paysans du Grand Nord Cameroun font généralement recours aux engrais chimiques de synthèse pour l'amélioration de la fertilité des sols. Or, l'usage des engrais chimiques, de par leur action bénéfique immédiate sur la productivité des cultures apporte une solution immédiate au problème de baisse de la fertilité (Temegne et al., 2017), mais leur coût élevé et leur indisponibilité les rendent presque inaccessibles aux petits paysans. En outre, son utilisation exclusive entraine une augmentation de l'acidité, une dégradation du statut physique et une baisse de la matière organique du sol (Mulaji, 2011). Selon les travaux de Claudine et al. (2013) sur le rendement et la conservation de l'oignon affectés par la fertilisation chimique et ceux de Habou et al. (2015) sur l'effet de l'azote sur l'aptitude à la conservation des bulbes d'oignon; des apports d'engrais azotés augmentent le rendement, réduisent le taux de matière sèche, accroissent la pourriture et la germination précoce, limitant leurs aptitudes à la conservation après la récolte. Les travaux de Ngonkeu et al. (2013) rapportent que l'utilisation des intrants par les agriculteurs est due à l'ignorance et la non exploitation des méthodes biologiques qui sont rentables et durables. Dans un tel contexte, la mise en place des pratiques agricoles à bas prix visant à augmenter la production agricole et reposant sur les respects des fonctionnalités écologiques est nécessaire (Nwaga et al., 2010 ; Megueni et al., 2011).

Les difficultés liées à la filière oignon dans le Grand Nord Cameroun corroborent en partie les données rencontrées dans la littérature. Plusieurs auteurs (PADFA, 2010 ; Mamadou, 2012 ; Folefack et Djouldé, 2013) rapportent que le manque de semences améliorées, les maladies et le transport du produit sont les problèmes majeurs des cultures maraîchères au Cameroun.

\section{Conclusion}

Les acteurs de la filière oignon sont pour la plupart des producteurs et ces derniers sont 1,47 fois plus nombreux que les commerçants. Quatre (04) variétés d'oignon sont cultivées dans la partie Septentrionale du Cameroun, il s'agit des variétés Goudami, Chagari, Belami et violet de Galmi. La variété Goudami avec un rendement en bulbe élevé (10 à $35 \mathrm{t} / \mathrm{ha}$ ) et une durée de conservation des bulbes plus importante est la plus cultivée. Les producteurs qui s'intéressent à la variété Goudami sont respectivement 4,91 fois ; 2,25 fois et 1,85 fois plus nombreux que les producteurs des variétés Belami, Chagari et Violet de Galmi. La quasi-totalité (69\%) des producteurs utilise les engrais chimiques pour améliorer le rendement de cette culture maraîchère. Ces informations serviront de base de données dans le programme de perfectionnement de la filière oignon au Cameroun. Nos études futures consisteront à améliorer de façon durable la disponibilité de l'oignon Cameroun à base des champignons mycorhiziens endogènes de la rhizosphère de cette culture maraîchère.

\section{CONFLIT D'INTÉRÊTS}

Les auteurs ont déclaré qu'il n'existe pas d'intérêts concurrents.

\section{CONTRIBUTIONS DES AUTEURS}

Ce travail a été réalisé avec la collaboration de tous les auteurs. CM et DN ont conçu l'étude et ont géré les recherches bibliographiques. LTT et AM ont rédigé le protocole et ont effectué les analyses statistiques. JD, H et HA ont mené les enquêtes et ont rédigé la première version du manuscrit. Tous les auteurs ont lu et approuvé la version finale du manuscrit.

\section{REMERCIEMENTS}

Les auteurs remercient les acteurs de la filière oignon de la partie Septentrionale du Cameroun pour leur hospitalité et le partage des connaissances. Ils remercient également les lecteurs anonymes pour leur contribution 
scientifique qui a permis d'améliorer qualité de ce manuscrit.

\section{RÉFÉRENCES}

Abdou R. 2014. Caractérisation de la diversité génétique de cultivars d'oignon (Allium cepa L.) du Niger en vue de leur conservation in situ et de leur amélioration. Thèse, Université de LiègeGembloux, 151p.

Abdou R, Bakasso Y, Adam T, Saadou M, Baudoin JP. 2014. Oignon (Allium cepa L) : biologie, système de culture et marqueurs génétiques pour l'analyse de la diversité en Afrique. Thèse, Université de Liège-Gembloux, Gembloux, 151p.

Assane DM. 2006. Les effets de la réappropriation de la culture du Violet de Galmi par les producteurs d'oignon de la région de Tahoua - NIGER, sur la dynamique du territoire local, l'organisation sociale et économique, Thèse de Doctorat, Option Développement Rural, Université de Toulouse-Le, Toulouse, $281 \mathrm{p}$.

Akoa J, Mabou A. 2004. La production de l'oignon dans la province de l'extrême nord du Cameroun : une activité génératrice de revenus. Agro-PME, Interdev Agroalimentaire, $5 \mathrm{p}$.

Bassole K, Dieye P, Durand A, Guerin J, Pioffret T. 2017. Amélioration de la rentabilité du système de culture de l'oignon, au Nord Cameroun. Ecole supérieur d'agro-développement international (ISTOM), $36 \mathrm{p}$.

Cathala M, Woin N, Essang T. 2003. L'oignon, une production en plein essor au NordCameroun. Cahier Agriculture, 12: 261266.

Claudine K, Paulo RE, Volnei P, Francisco OG. MJ, Joao VN. 2013. Yield and conservation of onions affected by nitrogen fertilization in no-tillage system. Horticultura Brasileira, 31(4): 559-567. DOI: https://dx.doi.org/10.1590/S0102

Folefack DP, Djouldé DR. 2013. Stratégies de commercialisation des produits maraichers sur le marché de Maroua, au Cameroun. Innovation Environnement Développement (IED) Afrique, 4p.

Fritsch RM, Friesen N. 2002. Evolution, domestication and taxonomy. In Allium Crop Science, Rabionwitch HD, Currah L (eds). Recent Advances CAB publishing: Oxon-Wallingford-UK; 5-30.

Habou ZA, Mahamadou CI, Adam T. 2015. Effet de l'azote sur l'aptitude à la conservation des bulbes d'oignon (Allium cepa L.). International Journal of Biological and Chemical Sciences, 9(6): 2889-2896. DOI: 10.4314/ijbcs.v9i6.28

Iyébi-Mandjek O. 2000. Cultures maraîchères au Nord Cameroun. In Notice 19: Atlas de la Province de l'Extrême Nord, IRD. IRD: Montpellier; 102-107.

Kamdem KF, Tchuenteu TL, Maimouna A, Megueni C. 2020. The combination of Arbuscular mycorrhizal Fungi with rock powder and poultry litter: an appropriate natural fertiliser for improving the productivity of soybean (glycine max (1.) merr). Agriculture, 66(3): 108-117. DOI: 10.2478/agri-2020-0010

Kamga RT, Tchouamo IR, Chendjou R, Bidogeza JC, Afari-Sefa V. 2016. Gender inequality in smallholder onion (Allium cepa 1.) production in the far north region of Cameroon. Journal of Gender, Agriculture and Food Security, 1(3): 85103. DOI: $10.19268 / J G A F S .132016 .5$

Konate M, Parkouda C, Tarpaga V, Guira F, Rouamba A, Sawadogo-Lingani H. 2018. Evaluation des potentialités nutritives et l'aptitude à la conservation de onze variétés d'oignon (Allium cepa $\mathrm{L}$.) bulbes introduits au Burkina Faso. International Journal of Biological and Chemical Sciences, 11(5): 2005-2015. DOI: 10.4314/ijbcs.v11i5.6

Madi A. 2000. Les prix des produits et le système productif dans la zone cotonnière de l'Extrême-Nord du Cameroun. Cahiers Agricultures, 9: 125-130.

Maldangoï Y, Woïn N, Cathala M. 2003. Les producteurs d'oignons au Nord 
Cameroun : les défis d'une filière à la quête d'une place au soleil. Actes du colloque Prasac Garoua : savanes africaines : des espaces en mutation, des acteurs face à de nouveaux défis, 27-30 mai $2002,8 \mathrm{p}$.

Mamadou BS. 2012. Appui à la commercialisation et à la structuration des filières riz et oignon et Stratégie opérationnelle pour la mise en œuvre des activités de la composante 2. PADFA II, Cameroun, 50p.

Megueni C, Awono Enama T, Ndjouenkeu R. 2011. Effet simultané de la dilution et de la combinaison du Rhizobium et des mycorhizes sur la production foliaire et les propriétés physico-chimiques des jeunes feuilles de Vigna unguiculata (L.) Walp. Journal of Applied Biosciences, 40: 2668-2676.

Mulaji KC. 2011. Utilisation des composts de biodéchets ménagers pour l'amélioration de la fertilité des sols acides de la province de Kinshasa (République Démocratique du Congo). Thèse de doctorat en sciences agronomiques et ingénierie biologique, Université de Liège- Gembloux Agro-Biotech, Gembloux, $220 \mathrm{p}$.

Ngonkeu MEL, Nwaga D, Adamou S, Chaintreuil C, The C, Fokom R, Amougou A, Tchameni NS, Onguene NA, Nana WL, Moulin L, Prin Y, Lebrun M, Dreyfus B. 2013. Diversité des champignons Mycorhiziens arbusculaires du Cameroun. Laboratoire des symbioses tropicales et méditerranéennes (LSTM), Campus International de Baillarguet, Montpellier, France, $15 \mathrm{p}$.
Nwaga D, Jansa J, Abossolo, Angue M, Frossard E. 2010. The potential of soil beneficial micro-organisms for slash-andburn agriculture in the humid forest zone of Sub-Saharan Africa. In Soil Biology and Agriculture in the Tropics, Dion (ed). Springer-Verlag: Berlin Heidelberg; 81107. DOI: http://dx.doi.org/10.1007/9783-642-05076-3.

Projet d'Appui au Développement des Filières Agricoles. 2010. PADFA, Rapport principal, République du Cameroun, $82 \mathrm{p}$.

Sali B, Olina JP, Adamou I. 2016. Compte rendu de mission de diagnostic des causes de pourritures des oignons. PADFA, Cameroun, $9 \mathrm{p}$.

Tache S, Ladam A, Corpet DE. 2007. Chemoprevention of aberrant crypt foci in the colon of rats by dietary onion. European Journal of Cancer, $\mathbf{4 3}(2)$ : 454458.

DOI:

https://dx.doi.org/10.1016/j.ejca.2006.09 .022

Temegne NC, Nkou FTD, Taffouo VD, Ntsomboh NG, Youmbi E. 2017. Influence of mycorrhization and phosphate fertilizer on growth of Voandzou (Vigna subterranea (L.) Verdc.). International Journal of Biological and Chemical Sciences, 11(6): 2587-2593.

DOI: https://dx.doi.org/10.4314/ijbcs.v11i6.3

Tchuenteu TL, Megueni C, Noubissié E, Wankoua Mana M. 2018. Study on physico-chemical properties of composts and their effects and growth, yield and fruits quality of Capsicum annum at Dang locality (Ngaoundéré Cameroon). Journal of Agriculture and Veterinary Science, 11(11): 26-35. DOI: 10.9790/2380-1111022635 